\title{
Interspecific complementation-restoration of phenotype in Arabidopsis cuc2cuc3 mutant by sugarcane CUC2 gene
}

Mohammad Aslam', Zeyuan She', Bello Hassan Jakada², Beenish Fakher², Joseph G. Greaves², Maokai Yan', Yingzhi Chen ${ }^{1}$, Ping Zheng ${ }^{2}$, Yan Cheng ${ }^{2}$ and Yuan Qin ${ }^{1,2^{*}}$

\begin{abstract}
Background: In plants, a critical balance between differentiation and proliferation of stem cells at the shoot apical meristem zone is essential for proper growth. The spatiotemporal regulation of some crucial genes dictates the formation of a boundary within and around budding organs. The boundary plays a pivotal role in distinguishing one tissue type from another and provides a defined shape to the organs at their developed stage. NAM/CUC subfamily of the NAC transcription factors control the boundary formation during meristematic development.

Results: Here, we have identified the CUP-SHAPED COTYLEDON (CUC) genes in sugarcane and named SsCUC2 (for the orthologous gene of CUC1 and CUC2) and SsCUC3. The phylogenetic reconstruction showed that SsCUCs occupy the CUC2 and CUC3 clade together with monocots, whereas eudicot CUC2 and CUC3 settled separately in the different clade. The structural analysis of CUC genes showed that most of the CUC3 genes were accompanied by an intron gain during eudicot divergence. Besides, the study of SsCUCs expression in the RNA-seq obtained during different stages of ovule development revealed that SsCUCS express in developing young tissues, and the expression of SsCUC2 is regulated by miR164. We also demonstrate that SsCUC2 (a monocot) could complement the cuc2cuc3 mutant phenotype of Arabidopsis (eudicot).

Conclusions: This study further supports that CUC2 has diverged in CUC1 and CUC2 during the evolution of monocots and eudicots from ancestral plants. The functional analysis of CUC expression patterns during sugarcane ovule development and ectopic expression of SsCUC2 in Arabidopsis showed that SsCUC2 has a conserved role in boundary formation. Overall, these findings improve our understanding of the functions of sugarcane CUC genes. Our results reveal the crucial functional role of CUC genes in sugarcane.
\end{abstract}

Keywords: Saccharum spontaneum, cuc2CUC3, organ boundary, CUP-SHAPED COTYLEDON (CUC), Complementationrestoration

*Correspondence: yuanqin001@foxmail.com

${ }^{2}$ Center for Genomics and Biotechnology, Fujian Provincial Key Laboratory of Haixia Applied Plant Systems Biology, 350002 Fuzhou, Fujian, China

Full list of author information is available at the end of the article

\section{Background}

In plants, different tissues and organs are produced in a specific pattern during embryonic and post-embryonic developments [1]. Shoot apical meristem (SAM) mainly constitutes the vegetative and floral meristem. Unlimited (or indeterminate) growth in vegetative tissues produces leaves and axillary branches at the nodes, whereas the limited (or determinate) growth is marked by the floral meristem where the SAM partitions its cells 
to divide in a highly coordinated manner to give rise to different floral parts [2]. The spatiotemporal differentiation of shoot apical cells forms specific patterns of meristems, initiated under tight regulation of boundary cells [3-9]. The regulatory network constitutes combinatory action of several elements, including transcription factors (TFs) [10]. The NAC transcription factors belong to one of the most prominent families of plant-specific TFs, constituting more than 100 members in Arabidopsis [11]. NACs derived their name from NAM (NO APICAL MERISTEM),ATAF 1/2 (Arabidopsistranscription activation factor) and CUC(CUP-SHAPED COTYLE$D O N)$ [12-14]. The members of NAC transcription factors, including CUC in Arabidopsis, NAM in Petunia hybrida and CUPULIFORMIS (CUP) in Antirrhinum majus, are responsible for boundary cell formation [1, 15, 16]. In Arabidopsis, three CUC genes, CUC1, CUC2 and $C U C 3$, are reported. $C U C 1$ and $C U C 2$ have diverged from each other through duplication event from a common ancestor. $C U C 2$ defines the shoot apical meristem zone, whereas CUC3 marks the boundary layer around developing organs. In general, primary shoot meristem development depends on CUCs activity, and axillary meristem requires mainly CUC2 and CUC3. Mutants of $C U C$ genes revealed various exclusive and partially overlapping phenotypes in different plant development processes such as SAM establishment, lateral organ separation, leaf serration, and ovule formation [1, 17-19]. These mutant phenotypes suggest that CUCs are necessary for normal ovule development and $\mathrm{CUCS}$ have higher expression in sporogenous tissue of ovule [20-31]. The low expression of CUCs decreases the number of leaflets causing fewer serrated leaves [10, 18, 32-34]. The expression pattern of $\mathrm{CUCs}$ is often synchronous and redundant in many cases; therefore, it becomes difficult to recognize a single knockout mutation. For example, defects in shoot apical meristem and cotyledon shapes can only be detectable under double and triple mutation of CUCs in Arabidopsis [17].

Previous findings indicate that Arabidopsis CUC1, $C U C 2$ and rice $C U C 1$ are post-transcriptionally targeted by miR164 $[18,35,36]$. This regulatory mechanism controls the balance between tissue separation/fusion and plays essential roles during leaf serration, phyllotaxy and growth $[18,37]$. Consistently, the plants display pronounced serrations, extra petals, and enlarged boundary domains when they express a miR164-resistant version of $C U C 1$ or $C U C 2$ [35]. Intriguingly, the role of the miR164CUC module in the regulation of leaf and floral organ morphology seems conserved across species [19, 37]. Besides miRNA, CUC gene expression is also reported to be regulated by chromatin remodeling [38]. Two SWI/SNF ATPases, AtBRM and SYD, are necessary for cotyledon separation in Arabidopsis by modulating the expression of $C U C$ genes [38].

Saccharum spp. (sugarcane), a tropical grass belonging to the family Poaceae is cultivated worldwide as a crop for sugar and biofuel feedstock. Sugarcane provides approximately $80 \%$ of sugar and $40 \%$ of bioethanol [39]. In terms of tonnage, it is the most cultivated crop than other staple crops (rice and wheat) (FAO, UN). Generally, the commercial cultivars are planted using stem cuttings with approximately 3-4 axillary buds that form primary shoots and roots after planting [40]. Due to the increasing demand for biofuel, researchers are putting continuous effort into improving sugarcane varieties for more sugar content while maintaining their hardiness. Recently, the sugarcane genome, one of the most complex genomes among all the crops, has been published, allowing researchers to study and improve the commercial varieties [41]. Here, we performed a genome-wide identification and expression analysis of $C U C$ genes from sugarcane to classify and better understand their functions. We found two CUC, SsCUC2 and SsCUC3 genes, in the sugarcane genome with unique expression patterns. We also found that the expression level of $\mathrm{SsCUC2}$ is regulated by miR164, which could be essential for sugarcane ovule development. Besides, we discovered that SsCUC2 could complement the cotyledon fusion and axillary meristem defects of the Arabidopsis cuc2cuc3 mutant. Despite the divergence of monocots and eudicots during evolution, the complementation of CUC2 from sugarcane (monocot) in Arabidopsis (eudicot) suggests a conserved role of $S s C U C 2$. Our results indicate that SsCUCs play a central role in sugarcane development, and miR164-SsCUC2 module could be essential for sugarcane ovule development.

\section{Results}

\section{Identification of the CUC genes in S. spontaneum}

A total of five sugarcane CUC gene sequences (three CUC2 alleles and two CUC3 alleles) were identified in the $S$. spontaneum genome (Table 1 ). The distribution of these $S s C U C$ genes was on chromosomes 6 and 7. The SsCUC proteins were 311-401 amino acid (aa) residues in length, where CUC2 alleles had 397, 400 and 401 aa residues, and CUC3 alleles were with 311 and 331 aa residues, respectively (Table 1 ). The molecular weight (MW) for the SsCUC proteins ranged from $34.198 \mathrm{kDa}$ to $42.251 \mathrm{kDa}$, and their isoelectric points (pI) varied from 7.22 to 9.03 . Additional parameters for sugarcane SsCUC proteins such as subcellular locations, N-glycosylation sites, and phosphorylation sites have also been predicted in this study. Based on the previous reports, the SsCUC proteins were expected for their localization in the nucleus, suggesting that they participate in gene 
Table 1 The physicochemical properties of CUC sequences in S. spontaneum and Arabidopsis thaliana

\begin{tabular}{llllllllll}
\hline & Name & Gene ID & A & B & C & $\begin{array}{l}\text { Amino acid } \\
\text { length (aa) }\end{array}$ & MW (kDa) & $\begin{array}{l}\text { Isoelectric } \\
\text { point }\end{array}$ & Chromosome position \\
\hline 1 & SsCUC2 & Sspon.07G0020380-1A & 0 & 4 & 2 & 401 & 42.251 & 8.76 & Chr7A:75915862-75918375 \\
2 & SsCUC2 & Sspon.07G0020380-2C & 0 & 4 & 2 & 400 & 42.107 & 8.9 & Chr7C:70928956-70931222 \\
3 & SsCUC2 & Sspon.07G0020380-3D & 0 & 4 & 3 & 397 & 41.452 & 9.03 & Chr7D:67919120-67921641 \\
4 & SsCUC3 & Sspon.06g0001780-1A & 1 & 3 & 0 & 331 & 36.215 & 7.22 & Chr6A:5729607-5731660 \\
5 & SsCUC3 & Sspon.06g0001780-2B & 1 & 3 & 0 & 311 & 34.198 & 8.52 & Chr6B:3987180-3989885 \\
6 & AtCUC1 & AT3G15170 & 3 & 4 & 1 & 310 & 34.232 & 8.58 & Chr3:5109782-5111608 \\
7 & AtCUC2 & AT5G53950 & 3 & 5 & 2 & 375 & 41.434 & 8.52 & Chr5:21901704-21903854 \\
8 & AtCUC3 & AT1G76420 & 3 & 3 & 3 & 334 & 38.017 & 6.46 & Chr1:28671806-28674045 \\
\hline
\end{tabular}

note:

' $A$ ' represents the number of $\mathrm{N}$-Glycosylation site

' $B$ ' represents the number of Protein kinase $C$ phosphorylation site in the NAM domain of the protein

' $C$ ' represents the number of Protein kinase $C$ phosphorylation site outside the NAM domain of the protein

Alleles are labeled with $-\mathrm{nX}$, where $\mathrm{n}$ represents alleles and $\mathrm{X}$ indicates haplotype ID, ranging from $A$ to $D$

expression regulation [42]. Interestingly, $\mathrm{N}$-glycosylation sites were not present in SsCUC2, whereas both the SsCUC3 had one N-glycosylation site at their C terminal end. Although both SsCUC2 and SsCUC3 had phosphorylation sites in the NAM domain, SsCUC2 possessed extra phosphorylation sites in the NAM domain. Besides, SsCUC3 did not own any phosphorylation sites outside the NAM domain whereas, SsCUC2 had 2 to 3 extra phosphorylation sites outside the NAM domain in the C-terminal region (Table -1, Additional File S1).

\section{Gene structure and motif composition of CUC genes}

The intron/exon organization and conserved motifs of the CUC genes from selected monocots and eudicots, including S. spontaneum, were studied to explore the structural features of $C U C$ genes and proteins encoded by the CUC genes. We identified ten different motifs in CUC proteins (Fig. 1). Among the 10 identified motifs, SsCUC2 had 8 motifs and SsCUC3 only had 6 motifs. Motif no. 8 and 10 were not present in the SsCUC2 whereas, motifs 6, 7, 8 and 9 were absent from SsCUC3 (Fig. 1). All the CUC3 proteins except SICUC3 had motif 10 , indicating that it could be CUC3 proteins specific motif. Motifs 7 and 9 were specific to CUC1 and CUC2 (Fig. 1). Motifs 1 to 5 were present in all the selected CUC proteins and represented the NAM domain.
The exon-intron organization of all of these $C U C$ genes was scanned to gain more insights into the CUC gene evolution. In general, the $C U C$ genes were intron-poor, and the number of introns varied from 1 to 3 for them. All the selected $C U C 1$ genes contained two introns, and most of the $C U C 2$ genes also had two introns except for $B d C U C 2$ and $O s C U C 2$, those possessed only one intron (Fig. 2). All the selected monocot CUC3 had only one intron except for the pineapple CUC3 (AcCUC3), which had two introns indicating an intron gain during evolution. At the same time, all the eudicot CUC3 had two introns except for the Eucalyptus grandis CUC3 (EgCUC3), which had 3 introns (Fig. 2). Additionally, two genes (AtCUC2 and AtCUC3) only had 5' untranslated region (UTR), and three genes (SlCUC1/2 and OsCUC3) had only 3' UTR whereas, 17 genes did not have any UTR. Altogether, these results suggest structural diversity among CUC genes (Fig. 2).

\section{Phylogenetic analysis of CUC proteins}

The evolution of CUC orthologs in different plant species was investigated by constructing a phylogenetic tree consisting of 39 CUC proteins from six monocots, including S. spontaneum and nine eudicots using the NeighborJoining (NJ) method (Fig. 3; Additional File S2). All the monocot CUC proteins were divided into two major groups, where one group represented monocot specific

(See figure on next page.)

Fig. 1 Schematic diagram representing the architecture and motif composition CUC genes in 6 monocots (Ss-Saccharum spontaneum, AC-Ananas comosus, Os-Oryza sativa, Bd-Brachypodium distachyon, Hv-Hordeum vulgare and Zm-Zea mays) and 9 eudicots (At-Arabidopsis thaliana, Br-Brassica rapa, Dc-Daucus carota, SI-Solanum lycopersicum, Eg-Eucalyptus grandis, Cs-Citrus sinensis, Gr-Gossypium raimondi, Gm-Glycine max and Pv-Phaseolus vulgaris). The motifs, numbers 1-10, are displayed in different colored boxes and detailed below 


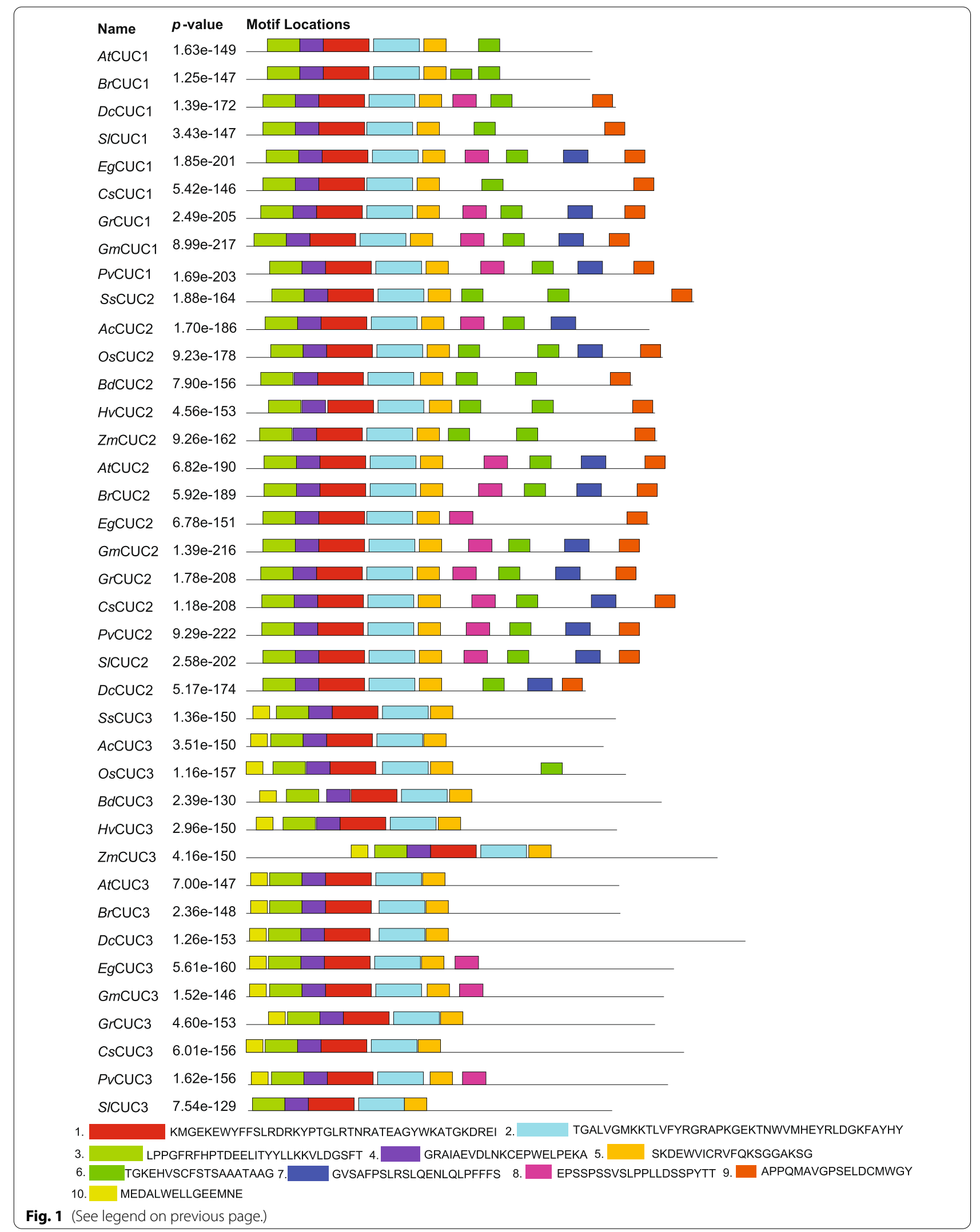




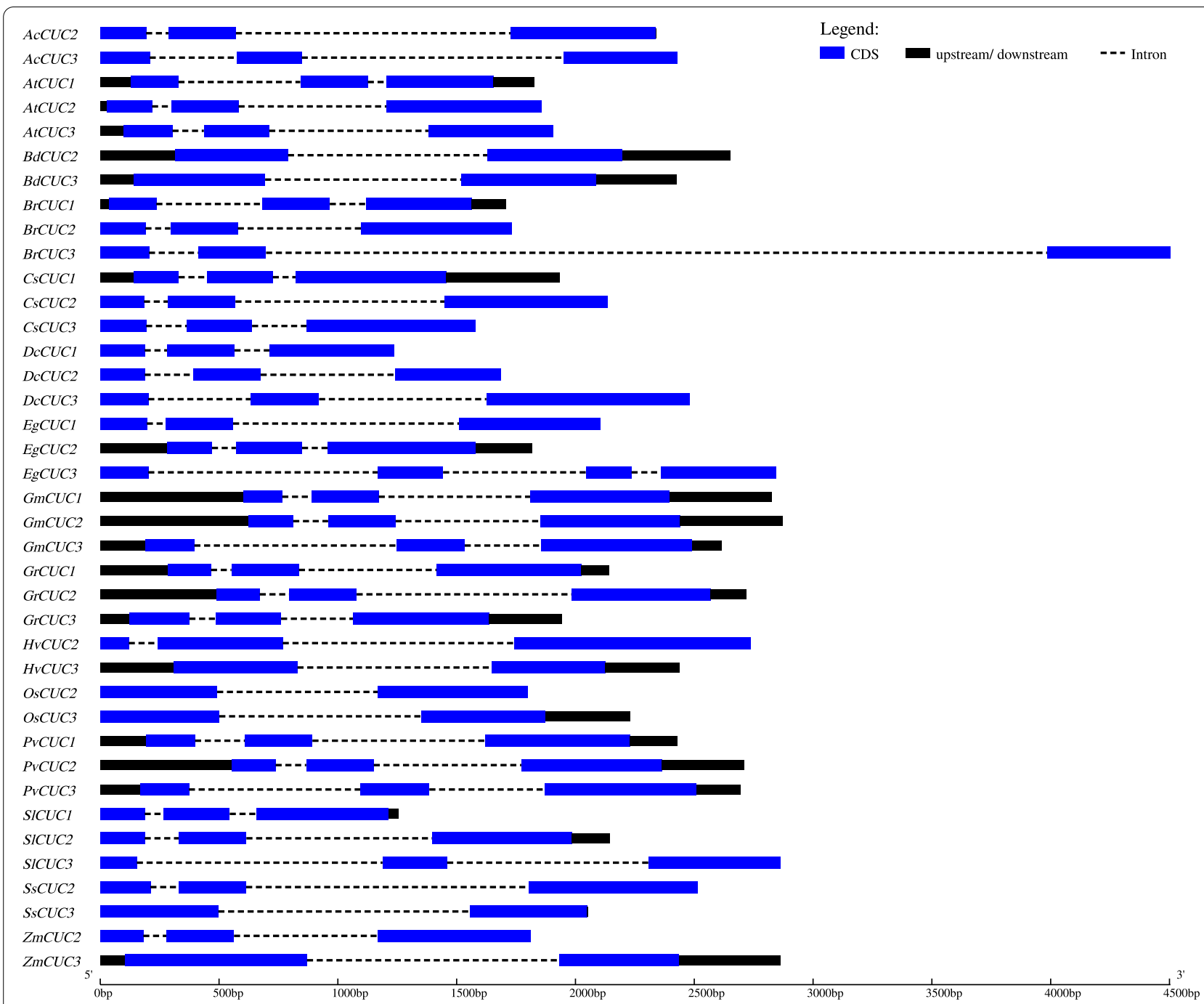

Fig. 2 Exon-intron structure of sugarcane SsCUC2, SsCUC3 and their selected homologous genes. Exons are represented as blue boxes, intron as dotted lines, and UTR are represented with black boxes. The different species are indicated as: Ss-Saccharum spontaneum, Ac-Ananas comosus, Os-Oryza sativa, Bd-Brachypodium distachyon, Hv-Hordeum vulgare, Zm-Zea mays, At-Arabidopsis thaliana, Br-Brassica rapa, Dc-Daucus carota, SI-Solanum lycopersicum, Eg-Eucalyptus grandis, Cs-Citrus sinensis, Gr-Gossypium raimondi, Gm-Glycine max and Pv-Phaseolus vulgaris

CUC2 and the other group represented monocot specific CUC3 proteins (Fig. 3). The eudicot CUC proteins were separated from monocots and grouped along with eudicot-specific CUCs. For example, eudicot CUC3 made a separate group with dicot-specific CUC3 proteins; however, it was still separated from eudicot CUC1 and CUC2 (Fig. 3).

\section{SsCUC2 has transcriptional activation ability}

The NAC family genes are highly conserved in plants, and it is involved in transcriptional regulation of many genes [11]. We used a GAL4-responsive reporter system to conduct a transient expression assay in yeast cells to verify the transcription activation feature of SsCUC2. Transformed yeast cells with the positive control (pGBKT7-53 + pGADT7-T) and (pGBKT7SsCUC2) grew well on synthetic dropout medium without tryptophan, histidine and leucine [SD (-Trp/His/-Leu)] and ensured $\alpha$-galactosidase ( $\alpha$-gal) activity. Yeast cells with empty pGBKT7 (negative control) exhibited no growth and $\alpha$-gal activity, indicating that SsCUC2 performs as transcription factors (Fig. 4 A). In addition, to determine the subcellular location of SsCUC2, we used $35 \mathrm{~S}: S s C U C 2-G F P$ fusion construct and transiently expressed the construct in $N$. benthamiana leaves. The results showed that SsCUC2 gets localized to the nucleus (Fig. 4B). 


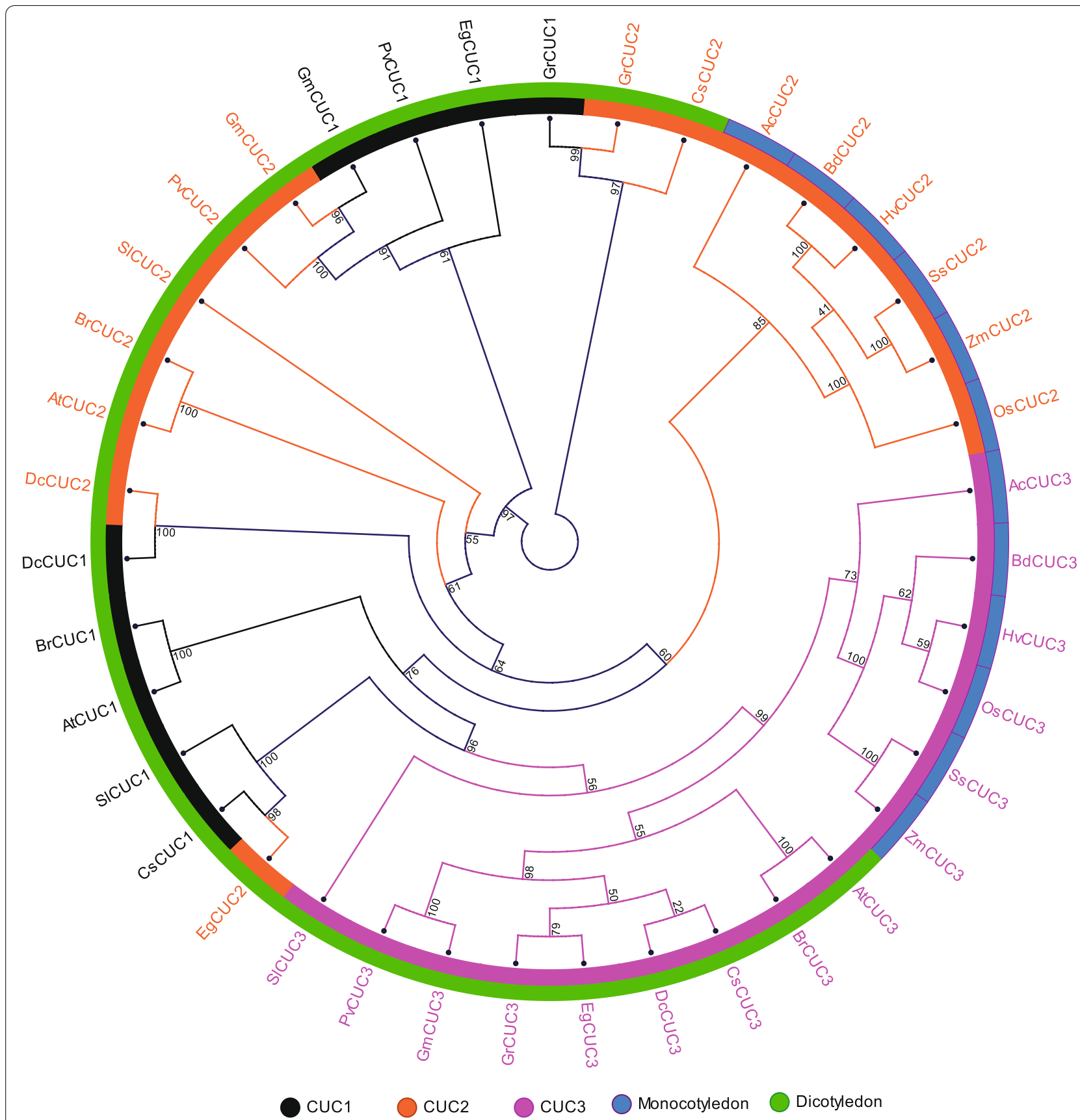

Fig. 3 The neighbor-joining phylogenetic tree analysis of sugarcane SsCUC2, SsCUC3 and their homologous from 5 monocots (Ac-Ananas comosus, Os-Oryza sativa, Bd-Brachypodium distachyon, Hv-Hordeum vulgare and Zm-Zea mays) and 9 eudicots (At-Arabidopsis thaliana, Br-Brassica rapa, Dc-Daucus carota, SI-Solanum lycopersicum, Eg-Eucalyptus grandis, Cs-Citrus sinensis, Gr-Gossypium raimondi, Gm-Glycine max and Pv-Phaseolus vulgaris). The tree was generated with a bootstrap value of 1000 generated in CLC sequence genomics workbench v12. CUC1 proteins are represented with black color, CUC2 proteins are represented with orange color and CUC3 proteins are represented with pink color. The sequence information of all the proteins used in the analysis is provided in Additional file S2

The expression of $S_{s} \mathrm{CUC} 2$ and $S s C U C 3$ is differentially regulated during sugarcane female gametophyte development

Previous research reports have implicated CUC1 and CUC2 in ovule formation and development, besides the mutant of $\mathrm{CUC1}$ and $\mathrm{CUC} 2$ results in the reduction of ovule number [43, 44]. During ovule development, the expression of CUC genes has been observed in medial, placental tissues 


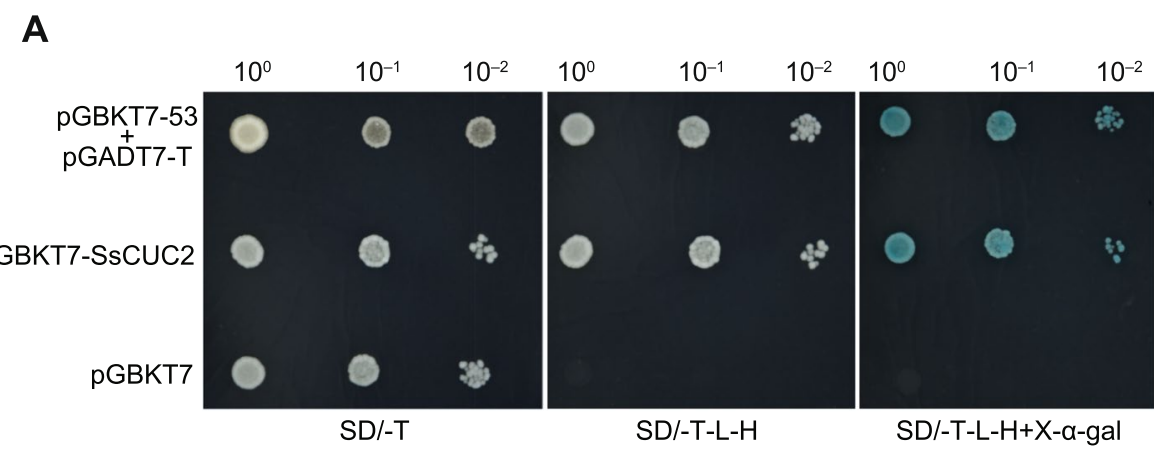

B
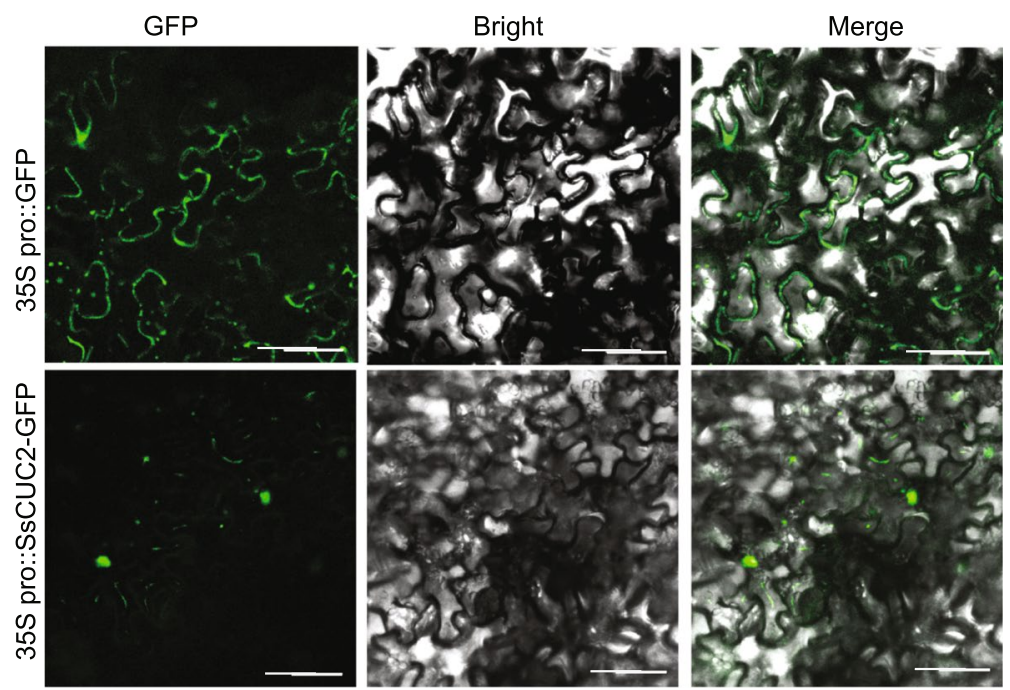

Fig. 4 A Transcription activity assay of SsCUC2. The ORF of SsCUC2 was introduced into the yeast expression vector pGBKT7. Yeast cells cotransformed with pGBKT7-53 + pGADT7-T were used as the positive control, and yeast cells with empty vector pGBKT7 were used as a negative control. The yeast cultures harboring respective vectors were grown on the synthetic medium supplied with dextrose (SD) in the absence of Trp (SD/ - T, left panel), on SD medium in the absence of Leu, Trp, and His (SD/ - L - T - H, middle panel) and on SD medium with a-galactosidase and in the absence of Leu, Trp, and His (SD/-L-T-H+X-a-gal, right panel). Yeast cells were incubated until $\mathrm{OD}_{600}$ reached 1 and then diluted 10 - and 100-fold for assays. B Subcellular localization of SsCUC2 in tobacco epidermal cells (lower panel)

and between the region of outgrowing ovules in Arabidopsis [44]. Therefore we analyzed the RNA-seq from different stages of sugarcane ovule development to study the expression patterns of $\mathrm{SsCUC2}$ and $\mathrm{SsCUC3}$. Consistent with previous reports, we found the differential expression of $S s C U C 2$ and $S s C U C 3$ during the sugarcane ovule development (Fig. 5 A). The expression of both the $S_{s} C U C 2$ and SsCUC3 increased tremendously at the megaspore mother cell (MMC) stage, which gradually decreased in the meiosis and mitosis stages. The expression of SsCUC2 and SsCUC3 return to approximately basal level at the mature stage of ovule development (Fig. 5 A). The differential expression patterns of SsCUC2 and SsCUC3 indicate that the CUC genes might also regulate the sugarcane ovule development.

\section{miR164a negatively regulates SsCUC2}

Previously, it has been demonstrated that miR164 posttranscriptionally regulates the CUC1 and CUC2 [18,
36, 45]. Therefore, we first checked whether SsCUC2 ( the orthologous gene of CUC1 and CUC2) expression is regulated by microRNA. Consequently, we investigated the corresponding miRNA using $\mathrm{SsCUC2}$ as a target in the psRNATarget database (http://plant grn.noble.org/psRNATarget/home) [46]. The results indicated that the microRNA miR164a might regulate $S_{s C U C 2}$ (Fig. 5B), which could also be regulating SsCUC2 during ovule development. Hence we checked the expression of miR164a in sRNA-seq from different stages of sugarcane ovule development. The sRNA-seq result suggested that the sugarcane miR164a was differentially accumulated in the developing sugarcane ovule (Fig. 5 A). The expression pattern of miR164a was initially decreased in the MMC stage and gradually increased in meiosis and mitosis stages, but the expression of miR164 exponentially increased at the mature stage (Fig. 5 A). The expression pattern of 

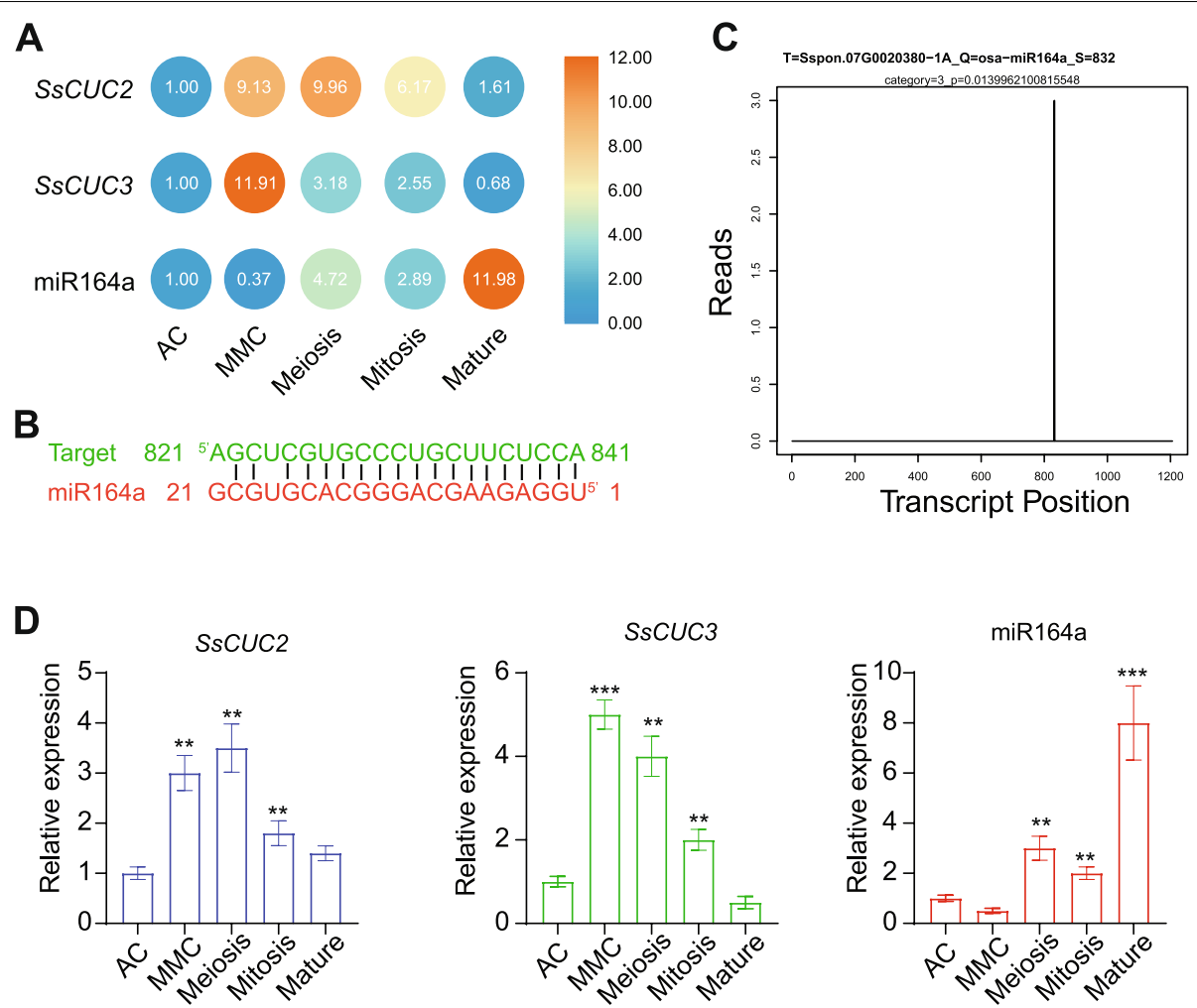

Fig. 5 Expression and regulation of sugarcane CUC genes. A The expression patterns of SsCUC2, SsCUC3 and miR164a in different developmental stages of ovules. The expression level for the AC stage was set to 1.0, and the data presented in MMC, Meiosis, Mitosis and Mature is relative to AC. B Predicted target site in SsCUC2 by psRNATarget database. C Target plot (T-plot) indicating the cleavage event mediated by miR164 on the PARE-seq. D RT-qPCR of SsCUC2, SsCUC3 and miR164a in different developmental stages of ovules. Sugarcane $\beta$-actin was used as an internal control. The expression level was set to 1.0 (for AC), and the data presented relative transcript abundance to AC. The threshold cycle (CT) values were used to calculate relative expression using the $2^{-\Delta \Delta C T}$ method. The data represent the means \pm SE of three replicates, and asterisks denote statistically significant values $\left({ }^{* *} p<0.01\right.$ and $\left.{ }^{* * *} p<0.001\right)$

miR164a in developing ovules endorses that it could be regulating the $S s C U C 2$ expression during ovule development. We further confirmed that miRNA164a cleaves the SsCUC2 using the PARE-seq generated (Fig. 5 C). We then checked the consistency of deep sequencing and validated the results using RT-qPCR. RT-qPCR results showed a similar trend to that of the sequencing data (Fig. 5D).

\section{SsCUC2 has a conserved function in meristem/organ boundary specification}

In arabidopsis, the double mutants of the CUC gene show defects in SAM formation and form cup-shaped cotyledon due to the fusion of cotyledons. However, the single mutants of any CUC genes have no significant boundary formation defects [1] (Additional File S3). To investigate the conserved function of CUC genes and check whether the SsCUC2 can rescue the phenotypic defects of cuc2cuc3 mutant, we ectopically expressed the $\mathrm{SsCUC2}$ in the Arabidopsis cuc2cuc3 mutant. During the transgenic screening on the hygromycin-based selection, we found that the plants carrying the sugarcane CUC2 gene grew bigger with two separate cotyledons and complemented the cup phenotype of cuc2cuc3 mutant (Fig. $6 \mathrm{~A}$ and 6B). The complemented lines (com1 and com2) showed normal plant growth with no SAM defects and two separate cotyledons in the next generation. These complemented plants showed a complete rescue of mutant phenotype during early vegetative and reproductive growth stages (Fig. $6 \mathrm{C}$ and D). Taken together, the results presented here suggest $S s C U C 2$ has a conserved role in boundary formation and early SAM formation.

\section{Discussion}

Plant-specific CUC TFs are required for several developmental progressions, such as establishing boundaries, the emergence of leaf primordia from apical meristem, floral organ separation, etc. $[1,10,15,47]$. However, the CUC genes have not been functionally characterized in monocots except in rice [48]. 

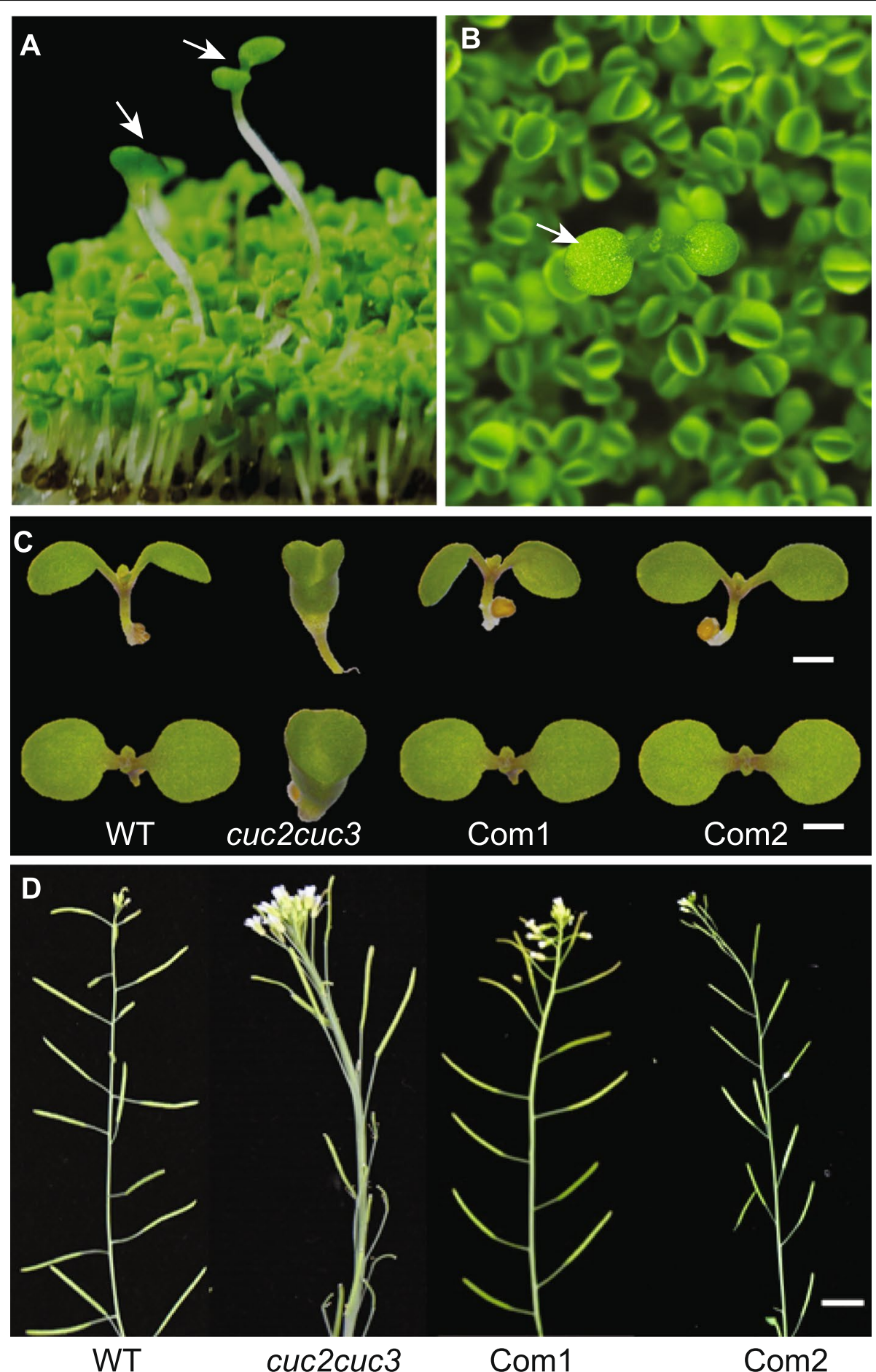

Fig. 6 Functional characterization of sugarcane CUC2 gene. A\&B) Hygromycin-based screening of $T 1$ transgenic plants. The plants carrying the SsCUC2 gene show normal two separate cotyledons (marked with white arrows), whereas the non-transformed with the cup-shaped fused cotyledon. Two complemented T2 lines (Com1 and Com2) showing the rescue of mutant phenotype C) at the early developmental stage with two separate cotyledons D) at the reproductive stage with normal inflorescences 
In this study, the $C U C$ genes from sugarcane were studied in detail. The identified $S s C U C$ genes were distributed on Chromosomes 7 A, 7 C, 7D (for SsCUC2), 6 A and 6B (for $\mathrm{SsCUC3}$ ). There is enough evidence to suggest that exon-intron structure variation is critical for the evolution of gene families. The gain and loss of exon-introns are caused by the reorganization and combination of different chromosome fragments [49]. The exon-intron organization study of the sugarcane $C U C$ genes and $C U C$ genes for 5 monocots and 6 eudicots indicated that $C U C$ genes are intron poor. Generally, the gene belonging to the same group shared similar intron-exon patterns with few exceptions (Fig. 2).

The similarities and differences among gene family proteins could be reflected in the composition of motifs [50]. The study of motifs present in CUC2 and CUC3 protein from different monocot and eudicot species revealed that most CUC2 proteins possess additional motifs compared to CUC3 (Fig. 1). Also, proper protein folding, activity and secretion of many proteins require $\mathrm{N}$-glycosylation, a common post-translational modification of proteins in eukaryotes. $\mathrm{N}$-linked glycans are processed in the ER by $\alpha$ glucosidases I and II and modified in the Golgi apparatus into complex structures [51-53]. Interestingly, among the five identified CUC sequences of sugarcane, only SsCUC3 sequences possessed an $\mathrm{N}$-glycosylation site at the $\mathrm{C}$-terminal region. Also, there were extra protein phosphorylation sites in SsCUC2 compared to SsCUC3 in the NAM domain and in the $\mathrm{N}$-terminal region (Table 1, Additional file 1). Both the N-linked glycosylation and protein phosphorylation play a crucial role in the activity of the protein. These differences between SsCUC2 and SsCUC3 could be the origin of the difference between their function and regulation.

Previous investigations of the evolutionary origin of CUC proteins suggest that the diversification of CUC3 from the CUC1/ CUC2 occurred more than 150 million years ago [54, 55]. Hasson et al. (2011) suggested that CUC2 may have preserved much of the inherited function after duplication of an ancestral gene, whereas CUC1 may have diverged, with changes affecting both the regulatory and coding regions of the gene [10]. We reconstructed the CUC phylogeny to investigate the evolutionary differences between the sugarcane CUC proteins and selected monocots and eudicot CUC proteins. Consistent with previous reports, the eudicot CUC formed two different clades, one with CUC1/CUC2 and another with CUC3. The monocot and eudicot sequences were also settled separately in the phylogeny (Fig. 3).

The ovule in seed plants forms the female gametophyte, which is responsible for fertilization and seed development. The primordia of the ovule comprise three separate regions. In Arabidopsis, CUCs have been linked to ovule initiation, ovule number, and ovule individualization [43, 44, 56, 57]. Previously, CUC transcripts were detected at the boundaries between the chalazal region, nucellus, and ovules [20]. In the RNA-seq of sugarcane, we also noticed the differential expression of CUCs during different developmental stages of the ovule. During the initial stages of ovule development, the expression of $S s C U C$ genes increased several folds and gradually decreased, returning to the basal levels at ovule maturation (Fig. $5 \mathrm{~A}$ and D). These observations indicate that $S s C U C$ genes participate in ovule development, which is in agreement with the previously reported functions of the CUC gene [20, 43, 56, 57].

In general, miRNAs function as negative regulators of the genes that act post-transcriptionally $[58,59]$. Several NAC genes are also targeted by miRNAs that regulate their expression, including NAM proteins of many gymnosperms that possess characteristic miR164 binding sites [19]. MIR164 regulates floral organ number and boundary development by creating and controlling the boundary domain by post-transcriptionally regulating CUC1 and CUC2 [35, 45]. In Arabidopsis, miR164-CUC2 module activity in the meristem tightly regulates plant development $[36,60,61]$. Mutations in the OsNAM, rice orthologous gene of CUC1 and CUC2, display a small phenotype with fused leaf structure, small panicles, and defective floral organs [48]. Also, overexpression of OsmiR164b displays a phenotype similar to OsNAM, and the OsmiR164-resistant version of OsNAM shows altered expression in the meristem, indicating that the OsNAM expression is regulated by OsmiR164 [36]. We also identified the miR164 binding site in sugarcane SsCUC2 using bioinformatics and PARE-seq data. Our results show that SsCUC2 possesses a miR164a binding site suggesting that the expression of sugarcane $\mathrm{CUC2}$ is also regulated by miR164a (Fig. 5B C).

Since CUCs have redundant functions in Arabidopsis, a single mutation does not induce an extreme phenotype, but the double mutation prevents the development of embryonic shoot meristems (Additional File S3) $[1,19]$. We verified the conserved function of the SsCUC2 gene by complementing the Arabidopsis cuc$2 c u c 3$ mutant phenotype using SsCUC2. The complemented plants rescued the cup phenotype of cuc2cuc3 mutant and distinctive SAM growth and floral architecture (Fig. 6 C \& D), suggesting that SsCUC2 has similar biological functions to AtCUC2.

\section{Conclusions}

Here we studied CUC genes in the sugarcane and explored their regulation during sugarcane ovule development. We also identified the conserved function of SsCUC2 in boundary formation and SAM development 
by ectopically expressing SsCUC2 in the Arabidopsis cuc$2 c u c 3$ mutant. Taken together, this study provides essential information about the conserved functions of the SsCUC2, especially in boundary formation and in ovule development, which is a critical trait in crop breeding.

\section{Methods}

Plant materials and growth condition and treatments Sugarcane (Saccharum officinarum L.) cultivar Yuetang 91-976 samples were collected from State Key Laboratory for Conservation and Utilization of Subtropical AgroBioresources, Guangxi, Nanning, China. DIC observation of the different stages of developing ovule [Archesporial cell (AC), MMC, meiosis, mitosis, and mature] was carried out to establish the developing stages in the sugarcane inflorescence, followed by sample collection for each stage using micro-dissection needles. All samples were snap-frozen in liquid nitrogen and stored in a deep freezer at $-80{ }^{\circ} \mathrm{C}$ for subsequent RNA extraction.

The Arabidopsis thaliana (Col-0; CS60000) was used as the wild-type, and all plants used were in Columbia background throughout this study. T-DNA mutants of cuc2-3, cuc3-105 and cuc2-3cuc3-105 were kindly provided by Dr. Nicolas Arnaud (INRA-AgroParisTech, France). Surface sterilized Arabidopsis seeds were placed in round, $90 \mathrm{~mm}$ Petri-plates on the modified Hoagland's medium containing $1 \%(\mathrm{w} / \mathrm{v})$ sucrose and $1 \%(\mathrm{w} / \mathrm{v})$ agar as described previously [59]. The plates were kept at $4{ }^{\circ} \mathrm{C}$ in the dark for 2 days for seed stratification. After stratification, the plates were transferred to the growth room at $22^{\circ} \mathrm{C}$ with a $16 \mathrm{~h}$ light $/ 8 \mathrm{~h}$ dark photoperiod under an intensity of $100 \mu \mathrm{mol} \mathrm{m}{ }^{-2} \mathrm{~s}^{-1}$, and seedlings were grown vertically [62]. For Arabidopsis transformation, Agrobacterium-mediated floral dip method was performed [63], and transgenic plants were selected on media plates containing $50 \mathrm{mg} \mathrm{l}^{-1}$ hygromycin.

\section{Identification of CUC genes}

The sugarcane (S. spontaneum) AP85-441 genome sequence data was downloaded from http://www.life.illin ois.edu/ming/downloads/Spontaneum_genome [41]. We searched CUC genes from the Saccharum genome using BLAST-P with the e-value set 0.01 with Arabidopsis CUC genes as the query. The identified sequences were further verified, and redundant sequences were removed. Besides, the CUC genes from 5 monocots (Ananas comosus, Oryza sativa, Brachypodium distachyon, Hordeum vulgare and Zea mays) and nine eudicots (Arabidopsis thaliana, Brassica rapa, Daucus carota, Solanum lycopersicum, Eucalyptus grandis, Citrus sinensis, Gossypium Raimondi, Glycine max, and Phaseolus vulgaris) were obtained from Phytozome V12.1 (https://phytozome.jgi. doe.gov) and NCBI (Additional file S2).

\section{Phylogenetic analysis}

The phylogenetic relationship of CUCs was studied using peptide sequences retrieved from monocots and eudicot plants listed above. The multiple sequence alignments were performed using MUSCLE, and the phylogenetic tree was constructed by CLC Genomics Workbench v12.0 (CLC Bio, Aarhus, Denmark) using the NeighborJoining method with default parameters and the bootstrap test of1000 replicates.

\section{Gene structure analysis and conserved Motif Identification}

The schematic CUCs structures were drawn by the Gene Structure Display Server 2.0 (http://gsds.gao-lab.org) [64]. The conserved motifs in the CUCs were identified by MEME (Multiple Em for Motif Elicitation) server 5.3.3 (https://meme-suite.org/meme) using default parameters with the maximum number of motifs set at 10 , and the optimal width of each motif was set between 6 and 100 residues.

\section{RNA-Seq, small RNA, and PARE-seq analysis}

RNA was extracted from the samples collected at different developmental stages of the ovule (AC, MMC, meiosis, mitosis, and mature) of the sugarcane. Ten mRNA-seq libraries, 10 small RNA-seq libraries (two replicate for each stage of developing ovule) and 5 degradome libraries were constructed (one replicate for each stage of ovule). Library construction and high-throughput RNA-seq, sRNA-seq, and PARE-seq were performed by LC Sciences (Zhejiang, China). After sequencing, the raw reads were filtered, and adapter sequences were removed along with contamination and low-quality reads from raw reads. The remaining unique sequences (clean reads) were then processed for further analysis. The transcript abundance of sugarcane CUC genes and miR164 was calculated as per million reads (RPM) method using CLC Genomics Workbench v12.0 (CLC Bio, Aarhus, Denmark). The heatmap was generated using TBtools after converting the expression values in fold change (additional file S4).

\section{RT-qPCR and expression analysis}

Total RNA was extracted using the RNeasy kit (Qiagen, MD, USA), followed by DNase I (Thermo Fisher Scientific, CA, USA) treatment. First-strand cDNA synthesis was carried out using 1ug of total RNA using ThermoScript RT-PCR kit (Thermo Fisher Scientific, CA, USA). In a CFX96 qPCR system (Bio-Rad, Singapore), quantitative PCR was performed with FastStart DNA Master SYBR Green I master mix (Takara, Shiga, Japan). Using $2^{-\Delta \Delta C T}$ method fold change in the expression was determined using the $\beta$-actin gene as the internal control. For miRNA, stem-loop qPCR was performed the reaction cycles were the same as with mRNA analysis. Three 
biological replicates and at least three separate technical replicates were used for each stage. Additional file S5 contains a list of the primers used in this study.

\section{Vector constructs}

The $S s$ CUC2-GFP was generated by amplifying the coding sequence of SsCUC2 (Sspon.07G0020380-1 A) without the stop codon from sugarcane leaf cDNA using the primers listed in additional file S5. The amplified PCR fragment was then cloned into the pENTR/D-TOPO vector (Invitrogen). pENTR/D-TOPO clones were then recombined into the destination vector pGWB505 using LR Clonase II (Invitrogen), and the construct was confirmed by sequencing.

\section{Transcriptional activation analysis in yeast cells}

The $S s C U C 2$ ORF was cloned into pGBKT7 to generate pGBKT7-SsCUC2 using the primers listed in additional file S5. The yeast strain AH109 was then transformed with pGBKT7, pGBKT7-53 + pGADT7-T, and pGBKT7SsCUC2. The transformed yeast cells were grown on $\mathrm{SD}$ (-Trp), SD (-Trp/-His/-Ade), and SD (-Trp/-His/-Ade/ $\alpha-$ gal). The growth status and $\alpha$-gal activity discovered the transactivation activity of $S s C U C$.

\section{Abbreviations}

NAM: NO APICAL MERISTEM; ATAF: Arabidopsis transcription activation factor ; CUC: CUP-SHAPED COTYLEDON; CUP: CUPULIFORMIS.

\section{Supplementary Information}

The online version contains supplementary material available at https://doi. org/10.1186/s12870-022-03440-z.

Additional file 1. Schematic diagram representing the NAM domain, $\mathrm{N}$-glycosylation sites and phosphorylation sites in SsCUC proteins. Red arrows represent the NAM domain, yellow arrowheads represent N-glycosylation sites and pink arrowheads represent phosphorylation sites.

Additional file 2. List of sequences used in the present study.

Additional file 3. Phenotype of CUC single mutants (cuc2, cuc3) and double mutant (cuc2cuc3).

Additional file 4. SsCUC expression and regulation. (A) A- Expression of CUC genes in RNA-seq, sRNA-seq and PARE-seq data of sugarcane during different stages of ovule development. (B) Normalized expression of miR164a in sRNA-seq data of sugarcane during different stages of ovule development. (C) Target identification of sugarcane CUC2 gene using degradome sequencing.

Additional file 5. List of primers used in the present study.

\section{Acknowledgements}

We thank all members of Qin lab for their assistance in the experiments.

\section{Authors' contributions}

MA and YQ conceived and designed the research. MA, ZS, BHJ, BF, JGG and MY performed the experiments. YC1, PZ and YC2 analyzed RNA-seq and sRNA-seq $M A, B F$ and $Y Q$ wrote the paper. All of the authors read and approved the final manuscript.

\section{Funding}

This work was supported by the Science and Technology Major Project of Guangxi (Gui Ke 2018-266-Z01) and Guangxi Distinguished Experts Fellowship to $Y Q$, postdoctoral project from Guangxi University to MA, and the National Natural Science Foundation of China (31970333). The funding bodies played no role in the design of the study and collection, analysis, and interpretation of data and in writing the manuscript.

\section{Availability of data and materials}

The sequencing data that support the findings of this study have been deposited in the NCBI SRA database with BioProject accession no. PRJNA723681, which will be available publicly upon acceptance of the article. All the protein and DNA sequences analyzed during this study are included in this article as Additional file S2. The expression values used to generate Fig. $5 \mathrm{~A}$ are provided in Additional file 54.

\section{Declarations}

Ethics approval and consent to participate

Not applicable.

\section{Consent for publication}

Not applicable.

\section{Competing interests}

The authors declare that they have no competing interests.

\section{Author details}

${ }^{1}$ Guangxi Key Lab of Sugarcane Biology, State Key Laboratory for Conservation and Utilization of Subtropical Agro-Bioresources, College of Agriculture, Guangxi University, 530004 Nanning, Guangxi, China. ${ }^{2}$ Center for Genomics and Biotechnology, Fujian Provincial Key Laboratory of Haixia Applied Plant Systems Biology, 350002 Fuzhou, Fujian, China.

Received: 17 April 2021 Accepted: 14 January 2022

Published online: 22 January 2022

\section{References}

1. Aida M, Ishida T, Fukaki H, Fujisawa H, Tasaka M. Genes involved in organ separation in Arabidopsis: an analysis of the cup-shaped cotyledon mutant. Plant Cell. 1997;9(6):841-57.

2. Fletcher JC. Shoot and floral meristem maintenance in arabidopsis. Annual Review of Plant Biology. 2002;53(1):45-66.

3. Aida M, Tasaka M. Morphogenesis and patterning at the organ boundaries in the higher plant shoot apex. Plant Mol Biol. 2006;60(6):915-28.

4. Tian C, Wang Y, Yu H, He J, Wang J, Shi B, Du Q, Provart NJ, Meyerowitz EM, Jiao Y. A gene expression map of shoot domains reveals regulatory mechanisms. Nature Communications. 2019;10(1):141.

5. Reinhart BJ, Liu T, Newell NR, Magnani E, Huang T, Kerstetter R, Michaels S, Barton MK. Establishing a framework for the Ad/abaxial regulatory network of Arabidopsis: ascertaining targets of class III homeodomain leucine zipper and KANADI regulation. Plant Cell. 2013;25(9):3228-49.

6. Wang Q, Hasson A, Rossmann S, Theres K. Divide et impera: boundaries shape the plant body and initiate new meristems. New Phytologist. 2016;209(2):485-98.

7. Hepworth SR, Pautot VA. Beyond the Divide: Boundaries for Patterning and Stem Cell Regulation in Plants. Front Plant Sci. 2015;6:1052.

8. Xu B, Li Z, Zhu Y, Wang H, Ma H, Dong A, Huang H. Arabidopsis genes AS1, AS2, and JAG negatively regulate boundary-specifying genes to promote sepal and petal development. Plant Physiol. 2008;146(2):566-75.

9. Lampugnani ER, Kilinc A, Smyth DR. PETAL LOSS is a boundary gene that inhibits growth between developing sepals in Arabidopsis thaliana. Plant J. 2012;71(5):724-35.

10. Hasson A, Plessis A, Blein T, Adroher B, Grigg S, Tsiantis M, Boudaoud A, Damerval C, Laufs P. Evolution and diverse roles of the CUP-SHAPED 
COTYLEDON genes in Arabidopsis leaf development. Plant Cell. 2011;23(1):54-68.

11. Ooka H, Satoh K, Doi K, Nagata T, Otomo Y, Murakami K, Matsubara K, Osato N, Kawai J, Carninci P, et al. Comprehensive analysis of NAC family genes in Oryza sativa and Arabidopsis thaliana. DNA Res. 2003;10(6):239-47.

12. Duval M, Hsieh TF, Kim SY, Thomas TL. Molecular characterization of AtNAM: a member of the Arabidopsis NAC domain superfamily. Plant Mol Biol. 2002;50(2):237-48.

13. Olsen AN, Ernst HA, Leggio LL, Skriver K. NAC transcription factors: structurally distinct, functionally diverse. Trends Plant Sci. 2005;10(2):79-87.

14. Aida M, Tasaka M. Genetic control of shoot organ boundaries. Curr Opin Plant Biol. 2006;9(1):72-7.

15. Weir I, Lu J, Cook H, Causier B, Schwarz-Sommer Z, Davies B. CUPULIFORMIS establishes lateral organ boundaries in Antirrhinum. Development. 2004;131(4):915-22.

16. Souer E, Houwelingen Av, Bliek M, Kloos D, Mol J, Koes R. Co-suppression of nam and homologous genes leads to a reduction in axillary meristem formation and increased leaf and stem size in Petunia: a possible role for NAC domain genes in plant development. Flowering Newsletter. 1998;26:36-46.

17. Vroemen CW, Mordhorst AP, Albrecht C, Kwaaitaal MA, de Vries SC. The CUP-SHAPED COTYLEDON3 gene is required for boundary and shoot meristem formation in Arabidopsis. Plant Cell. 2003;15(7):1563-77.

18. Nikovics K, Blein T, Peaucelle A, Ishida T, Morin H, Aida M, Laufs P. The balance between the MIR164A and CUC2 genes controls leaf margin serration in Arabidopsis. Plant Cell. 2006;18(11):2929-45.

19. Phillips HR, Landis JB, Specht CD. Revisiting floral fusion: the evolution and molecular basis of a developmental innovation. J Exp Bot. 2020;71(12):3390-404

20. Galbiati F, Sinha Roy D, Simonini S, Cucinotta M, Ceccato L, Cuesta C, Simaskova M, Benkova E, Kamiuchi Y, Aida M, et al. An integrative model of the control of ovule primordia formation. Plant J. 2013;76(3):446-55.

21. Raman S, Greb T, Peaucelle A, Blein T, Laufs P, Theres K. Interplay of miR164, CUP-SHAPED COTYLEDON genes and LATERAL SUPPRESSOR controls axillary meristem formation in Arabidopsis thaliana. Plant J. 2008;55(1):65-76.

22. Hibara K, Karim MR, Takada S, Taoka K, Furutani M, Aida M, Tasaka M. Arabidopsis CUP-SHAPED COTYLEDON3 regulates postembryonic shoot meristem and organ boundary formation. Plant Cell. 2006;18(11):2946-57.

23. Heisler MG, Ohno C, Das P, Sieber P, Reddy GV, Long JA, Meyerowitz EM. Patterns of auxin transport and gene expression during primordium development revealed by live imaging of the Arabidopsis inflorescence meristem. Curr Biol. 2005;15(21):1899-911.

24. Takada S, Hibara K, Ishida T, Tasaka M. The CUP-SHAPED COTYLEDON1 gene of Arabidopsis regulates shoot apical meristem formation. Development (Cambridge, England). 2001;128(7):1127-35.

25. Huang T, Harrar Y, Lin C, Reinhart B, Newell NR, Talavera-Rauh F, Hokin SA, Barton MK, Kerstetter RA. Arabidopsis KANADI1 acts as a transcriptional repressor by interacting with a specific cis-element and regulates auxin biosynthesis, transport, and signaling in opposition to HD-ZIPIII factors. Plant Cell. 2014:26(1):246-62.

26. Gonçalves B, Hasson A, Belcram K, Cortizo M, Morin H, Nikovics K, Vialette-Guiraud A, Takeda S, Aida M, Laufs P, et al. A conserved role for CUP-SHAPED COTYLEDON genes during ovule development. Plant J. 2015;83(4):732-42.

27. Cucinotta M, Manrique S, Cuesta C, Benkova E, Novak O, Colombo L. CUPSHAPED COTYLEDON1 (CUC1) and CUC2 regulate cytokinin homeostasis to determine ovule number in Arabidopsis. J Experimental Botany. 2018;69(21):5169-76.

28. Jameson PE, Song J. Cytokinin: a key driver of seed yield. J Exp Bot. 2016;67(3):593-606.

29. Ceccato L, Masiero S, Sinha Roy D, Bencivenga S, Roig-Villanova I, Ditengou FA, Palme K, Simon R, Colombo L. Maternal control of PIN1 is required for female gametophyte development in Arabidopsis. PLoS One. 2013;8(6):e66148.

30. Bencivenga S, Simonini S, Benkova E, Colombo L. The transcription factors BEL1 and SPL are required for cytokinin and auxin signaling during ovule development in Arabidopsis. Plant Cell. 2012;24(7):2886-97.
31. Yuan J, Kessler SA. A genome-wide association study reveals a novel regulator of ovule number and fertility in Arabidopsis thaliana. PLoS genetics. 2019;15(2):e1007934.

32. Blein T, Pulido A, Vialette-Guiraud A, Nikovics K, Morin H, Hay A, Johansen IE, Tsiantis M, Laufs P. A conserved molecular framework for compound leaf development. Science (New York, NY). 2008;322(5909):1835-9.

33. Bilsborough GD, Runions A, Barkoulas M, Jenkins HW, Hasson A, Galinha C, Laufs P, Hay A, Prusinkiewicz P, Tsiantis M. Model for the regulation of Arabidopsis thaliana leaf margin development. Proc Natl Acad Sci U S A. 2011;108(8):3424-9.

34. Blein T, Pautot V, Laufs P. Combinations of Mutations Sufficient to Alter Arabidopsis Leaf Dissection. Plants (Basel, Switzerland). 2013;2(2):230-47.

35. Laufs P, Peaucelle A, Morin $\mathrm{H}$, Traas J. MicroRNA regulation of the CUC genes is required for boundary size control in Arabidopsis meristems. Development. 2004;131(17):4311-22.

36. Wang J, Bao J, Zhou B, Li M, Li X, Jin J. The osa-miR164 target OsCUC1 functions redundantly with OsCUC3 in controlling rice meristem/organ boundary specification. New Phytol. 2021;229(3):1566-81.

37. Vialette-Guiraud AC, Adam H, Finet C, Jasinski S, Jouannic S, Scutt CP. Insights from ANA-grade angiosperms into the early evolution of CUPSHAPED COTYLEDON genes. Ann Bot. 2011;107(9):1511-9.

38. Kwon CS, Hibara K, Pfluger J, Bezhani S, Metha H, Aida M, Tasaka M, Wagner D. A role for chromatin remodeling in regulation of CUC gene expression in the Arabidopsis cotyledon boundary. Development. 2006;133(16):3223-30.

39. Su W, Huang L, Ling H, Mao H, Huang $N$, Su Y, Ren Y, Wang D, Xu L, Muhammad K, et al. Sugarcane calcineurin B-like (CBL) genes play important but versatile roles in regulation of responses to biotic and abiotic stresses. Sci Rep. 2020;10(1):167.

40. Ortiz-Morea FA, Vicentini R, Silva GF, Silva EM, Carrer H, Rodrigues AP, Nogueira FT. Global analysis of the sugarcane microtranscriptome reveals a unique composition of small RNAs associated with axillary bud outgrowth. J Exp Bot. 2013;64(8):2307-20.

41. Zhang J, Zhang X, Tang H, Zhang Q, Hua X, Ma X, Zhu F, Jones T, Zhu X, Bowers J, et al. Allele-defined genome of the autopolyploid sugarcane Saccharum spontaneum L. Nat Genet. 2018;50(11):1565-73.

42. Taoka K, Yanagimoto Y, Daimon Y, Hibara K, Aida M, Tasaka M. The NAC domain mediates functional specificity of CUP-SHAPED COTYLEDON proteins. Plant J. 2004;40(4):462-73.

43. Cucinotta M, Manrique S, Cuesta C, Benkova E, Novak O, Colombo L. CUP-SHAPED COTYLEDON1 (CUC1) and CUC2 regulate cytokinin homeostasis to determine ovule number in Arabidopsis. J Exp Bot. 2018;69(21):5169-76

44. Goncalves B, Hasson A, Belcram K, Cortizo M, Morin H, Nikovics K, Vialette-Guiraud A, Takeda S, Aida M, Laufs P, et al. A conserved role for CUP-SHAPED COTYLEDON genes during ovule development. Plant J. 2015:83(4):732-42.

45. Baker CC, Sieber P, Wellmer F, Meyerowitz EM. The early extra petals1 mutant uncovers a role for microRNA miR164c in regulating petal number in Arabidopsis. Curr Biol. 2005;15(4):303-15.

46. Dai X, Zhuang Z, Zhao PX. psRNATarget: a plant small RNA target analysis server (2017 release). Nucleic Acids Res. 2018;46(W1):W49-54.

47. Gonzalez-Carranza ZH, Zhang X, Peters JL, BoltzV, Szecsi J, Bendahmane M, Roberts JA. HAWAIIAN SKIRT controls size and floral organ number by modulating CUC1 and CUC2 expression. PLoS One. 2017;12(9):e0185106.

48. Chang Z, Xu R, Xun Q, Liu J, Zhong T, Ding Y, Ding C. OsmiR164-targeted OsNAM, a boundary gene, plays important roles in rice leaf and panicle development. Plant J. 2020;106(1):41-55.

49. Guo R, Xu X, Carole B, Li X, Gao M, Zheng Y, Wang X. Genome-wide identification, evolutionary and expression analysis of the aspartic protease gene superfamily in grape. BMC Genomics. 2013;14:554.

50. Su W, Ren Y, Wang D, Huang L, Fu X, Ling H, Su Y, Huang N, Tang H, Xu L, et al. New insights into the evolution and functional divergence of the CIPK gene family in Saccharum. BMC Genomics. 2020:21(1):868.

51. Marin-Menguiano M, Moreno-Sanchez I, Barrales RR, Fernandez-Alvarez A, Ibeas Jl. N-glycosylation of the protein disulfide isomerase Pdi1 ensures full Ustilago maydis virulence. PLoS Pathog. 2019;15(11):e1007687.

52. Ruddock LW, Molinari M. N-glycan processing in ER quality control. J Cell Sci. 2006;119(Pt 21):4373-80.

53. Lerouxel O, Mouille G, Andeme-Onzighi C, Bruyant MP, Seveno M, Loutelier-Bourhis C, Driouich A, Hofte H, Lerouge P. Mutants in DEFECTIVE 
GLYCOSYLATION, an Arabidopsis homolog of an oligosaccharyltransferase complex subunit, show protein underglycosylation and defects in cell differentiation and growth. Plant J. 2005;42(4):455-68.

54. Zimmermann R, Werr W. Pattern formation in the monocot embryo as revealed by NAM and CUC3 orthologues from Zea mays L. Plant Mol Biol. 2005;58(5):669-85.

55. Wikstrom N, Savolainen V, Chase MW. Evolution of the angiosperms: calibrating the family tree. Proc Biol Sci. 2001;268(1482):2211-20.

56. Ishida T, Aida M, Takada S, Tasaka M. Involvement of CUP-SHAPED COTYLEDON genes in gynoecium and ovule development in Arabidopsis thaliana. Plant Cell Physiol. 2000;41(1):60-7.

57. Cucinotta M, Cavalleri A, Guazzotti A, Astori C, Manrique S, Bombarely A, Oliveto S, Biffo S, Weijers D, Kater MM, et al. Alternative Splicing Generates a MONOPTEROS Isoform Required for Ovule Development. Curr Biol. 2020;31(4):892-9.

58. Voinnet O. Origin, biogenesis, and activity of plant microRNAs. Cell. 2009;136(4):669-87.

59. Aslam M, Sugita K, Qin Y, Rahman A. Aux/IAA14 Regulates microRNAMediated Cold Stress Response in Arabidopsis Roots. Int J Mol Sci. 2020:21(22):8441.

60. Peaucelle A, Morin H, Traas J, Laufs P. Plants expressing a miR164-resistant CUC2 gene reveal the importance of post-meristematic maintenance of phyllotaxy in Arabidopsis. Development. 2007;134(6):1045-50.

61. Sieber P, Gheyselinck J, Gross-Hardt R, Laux T, Grossniklaus U, Schneitz K. Pattern formation during early ovule development in Arabidopsis thaliana. Dev Biol. 2004;273(2):321-34.

62. Aslam M, Fakher B, Jakada BH, Zhao L, Cao S, Cheng Y, Qin Y. GenomeWide Identification and Expression Profiling of CBL-CIPK Gene Family in Pineapple (Ananas comosus) and the Role of AcCBL1 in Abiotic and Biotic Stress Response. Biomolecules. 2019:9(7):293.

63. Clough SJ, Bent AF. Floral dip: a simplified method for Agrobacterium-mediated transformation of Arabidopsis thaliana. Plant $\mathrm{J}$. 1998;16(6):735-43.

64. Hu B, Jin J, Guo AY, Zhang H, Luo J, Gao G. GSDS 2.0: an upgraded gene feature visualization server. Bioinformatics. 2015;31(8):1296-7.

\section{Publisher's Note}

Springer Nature remains neutral with regard to jurisdictional claims in published maps and institutional affiliations.

Ready to submit your research? Choose BMC and benefit from:

- fast, convenient online submission

- thorough peer review by experienced researchers in your field

- rapid publication on acceptance

- support for research data, including large and complex data types

- gold Open Access which fosters wider collaboration and increased citations

- maximum visibility for your research: over $100 \mathrm{M}$ website views per year

At BMC, research is always in progress.

Learn more biomedcentral.com/submissions 